(2) Open Access Full Text Article

\title{
Intraoperative optical coherence tomography and $a b$ interno trabecular meshwork surgery with the Trabectome
}

This article was published in the following Dove Press journal:

Clinical Ophthalmology

28 September 2017

Number of times this article has been viewed

\section{Bernd Junker' \\ Jens $F$ Jordan ${ }^{2}$ \\ Carsten Framme' \\ Amelie Pielen'}

'University Eye Hospital, Medical School Hannover, Hannover, ${ }^{2}$ Eye

Center, Medical Center, University of

Freiburg, Freiburg, Germany
Correspondence: Bernd Junker University Eye Hospital, Medical School

Hannover, Carl-Neuberg-Straße I, 30625

Hannover, Germany

Tel +495II 5323060

Email junker.bernd@mh-hannover.de
Importance: This study is the first description of the use of the intraoperative optical coherence tomography (iOCT) for trabecular meshwork surgery with the Trabectome in a regular clinical setting.

Background: The aim of this study is to evaluate intraoperatively the immediate success of $a b$ interno trabeculotomy with the Trabectome defined as a removal of the trabecular meshwork. Design: This is a retrospective clinical study performed in the University Eye Hospital, Medical School Hannover.

Participants: A total of nine consecutive Caucasian patients suffering from primary open angle glaucoma, pigment dispersion glaucoma, or pseudoexfoliation glaucoma took part in the study.

Methods: All patients underwent ab interno trabeculotomy surgery with the Trabectome using a commercially available iOCT to visualize the anterior chamber angle (ACA) before and after the procedure. The visualization was done using a modified Swan-Jacobs lens (all nine patients) or without lens (view from above, five patients).

Main outcome measures: The main outcome of this study is the success of visualization of the ACA on iOCT, especially the postprocedural visualization of the wound gap after removal of the trabecular meshwork.

Results: Using the view from above, the ACA could be visualized before and after the procedure in only two of the five cases. Using the modified Swan-Jacobs lens, the ACA could be visualized before the procedure and the trabecular meshwork opening after the procedure in all nine patients.

Conclusion: The iOCT can be used to objectify the immediate success of the surgical procedure, ie, the removal of the trabecular meshwork, of ab interno trabeculotomy with the Trabectome. The procedure itself cannot be captured sufficiently via iOCT.

Keywords: glaucoma, imaging, iOCT, trabecular meshwork surgery

\section{Introduction}

In glaucomatous eyes, the trabecular meshwork acts as an effective barrier against the outflow of aqueous humor. Removal of this tissue by ab interno trabeculotomy with the Trabectome (NeoMedix, Tustin, CA, USA) can effectively lower the intraocular pressure. ${ }^{1}$ A successful surgical procedure is presumed when the whitish scleral backwall of Schlemm's canal can be seen during the surgery. ${ }^{2}$ Since the view into the anterior chamber angle (ACA) is sometimes blurred due to corneal opacity or intraocular bleeding, an objective method to obtain control of success is desirable. Intraocular microendoscopy ${ }^{3}$ and ultrasound biomicroscopy ${ }^{4}$ have been investigated to achieve 
this goal, but their use was not beneficial. Intraoperative optical coherence tomography (iOCT) is a promising new technique that can help to visualize intraocular tissue during the surgical procedure. In glaucoma surgery, there are just a few published studies describing the use of an iOCT and none of them investigates the effect of ab interno trabeculotomy on the ACA. ${ }^{5-7}$ The aim of our study was to demonstrate the possibility to visualize the ACA and the trabecular meshwork intraoperatively and to monitor the procedure of ab interno trabeculotomy with the Trabectome by visualizing the ACA pre- and postprocedural using an iOCT.

\section{Methods}

\section{Ethics statement}

Approval from the Ethics Committee of the Medical School Hannover for this retrospective study was obtained (approval number 2717-2015). All procedures performed were in accordance with the 1964 Declaration of Helsinki and its later amendments or comparable ethical standards.

All nine consecutive Caucasian patients (five males and four females; age between 51 and 85 years) who underwent $\mathrm{ab}$ interno trabeculotomy with the Trabectome between June and July 2014 were included in the study. Surgery was done using the surgical microscope LUMERA 700 and the inbuilt iOCT RESCAN 700 (Carl Zeiss Meditec AG, Jena, Germany). All patients gave their informed consent for the surgery and for the use of imaging material. The underlying disease was primary open angle glaucoma, pigment dispersion glaucoma, or pseudoexfoliation glaucoma.

The RESCAN 700 is a new spectral domain OCT with a center wavelength of $840 \mathrm{~nm}$ and a scanning speed of 27,000 A-scans/second, which is integrated in a LUMERA 700 (Carl Zeiss Meditec AG) surgical microscope (OPMI). It has an A-scan depth of $2.0 \mathrm{~mm}$ and an axial resolution of $5.5 \mu \mathrm{m}$ in tissue. The scan length is adjustable from 3 to $16 \mathrm{~mm}$, and the scan can be rotated by $360^{\circ}$. The surgeon is in full control of iOCT and microscope functions via the foot control panel of the OPMI. Additionally, the iOCT can be controlled by an assistant via touchscreen display. iOCT and microscope images are displayed on the touchscreen and real-time iOCT images as well as captured iOCT images, and the scan marker can be merged and injected into one eyepiece of the OPMI as an overlay. The surgeon may simultaneously observe iOCT and live operation site.

Videos of the whole Trabectome surgeries were captured and analyzed. We tried to display the trabecular meshwork before, during, and after the surgical procedure. Surgery was performed individually by two surgeons (BJ and AP) under general anesthesia, which is standard in our hospital.
After a temporal $1.7 \mathrm{~mm}$ clear cornea incision, the Trabectome single-use handpiece was inserted into the anterior chamber and the ACA was visualized using the modified Swan-Jacob goniolens (Ocular Instruments, Bellevue, WA, USA). Removal of the trabecular meshwork of the chamber angle opposite the corneal incision was achieved with a power of $0.8 \mathrm{~W}$ over $90-120^{\circ}$. Retrograde bleeding from the collector channels was rinsed out using balanced salt solution (Beaver-Visitec International Inc., Waltham, MA, USA).

iOCT images of the nasal chamber angle were captured in only five of the nine patients using the direct view from above (before and after the ab interno trabeculotomy). In all patients, iOCT images were taken before, during, and after the surgical procedure using the modified Swan-Jacob lens with the Trabectome handpiece inside the anterior chamber and the patient's head rotated to the opposing side.

Surgical success was defined as a recognizable trabecular meshwork opening on the iOCT images.

\section{Results}

In all patients, we had a clear view of the ACA using the Swan-Jacob lens at the beginning of the procedure. At the end of the procedure, the presumed scleral backwall of Schlemm's canal could also be seen in all patients.

Using the view from above the trabecular meshwork is shown in two of the first five cases at the beginning of the surgery (Figure 1A). In the postprocedure images, we demonstrate the anterior part of the trabecular meshwork opening also in two of these five cases (Figure 1B). Sometimes, the reflux bleeding prevented the view temporarily (Figure 1C). Because of these disappointing results, we did not use the view from above in the following four patients.

Using the modified Swan-Jacob lens, we demonstrate the chamber angle in all nine cases. Best results were achieved using the shortest scan length of $3 \mathrm{~mm}$ and a vertical scan mode. Figure 2 shows examples of pre- and postprocedure images, the latter with an open wound gap. This wound gap is shown in all patients. It correlated to the area where we could see the presumed scleral backwall of Schlemm's canal. With the horizontal scan mode, we identify the trabecular meshwork opening only in two cases due to the difference in focus level (Figure 2C).

We could not capture images of the Trabectome tip inside Schlemm's canal or get sharp images or videos during the removal of the trabecular meshwork.

It took us between 2 and 15 minutes to obtain the iOCT images in addition to the regular surgical time (15 minutes for the first two patients and between 2 and 4 minutes for the last four patients). 
A
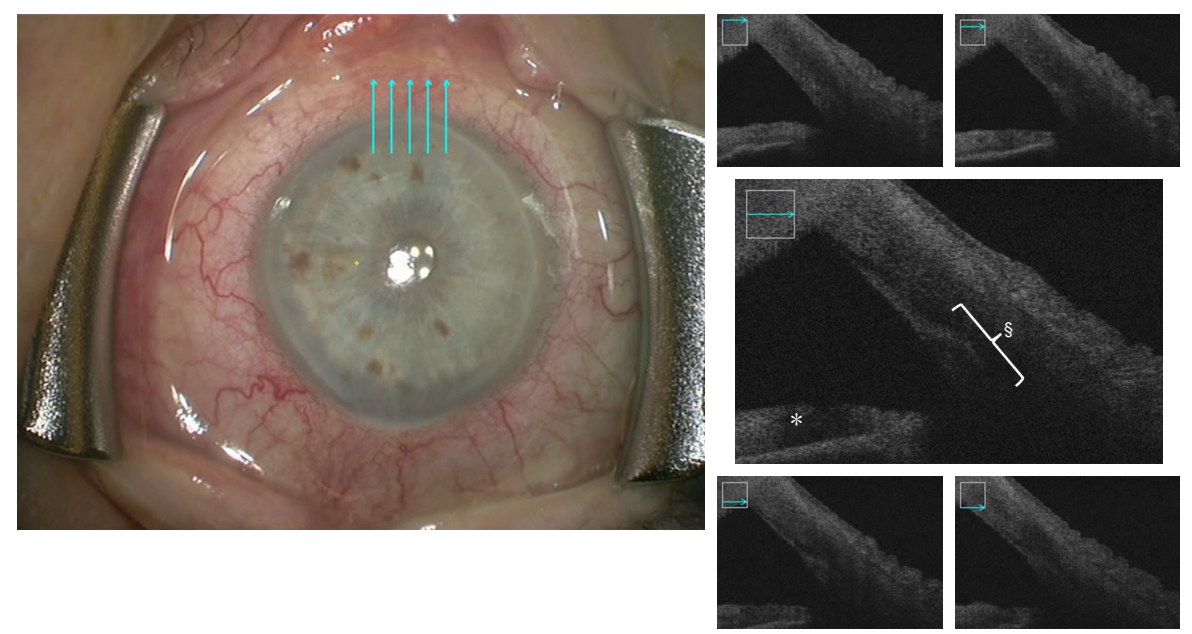

B
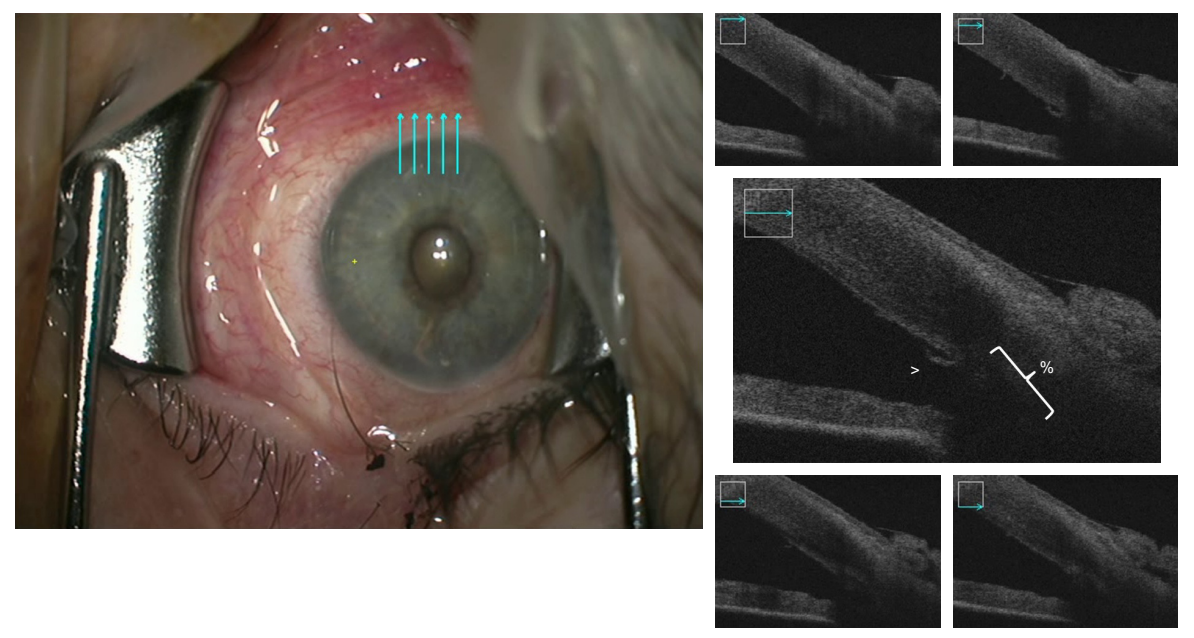

C
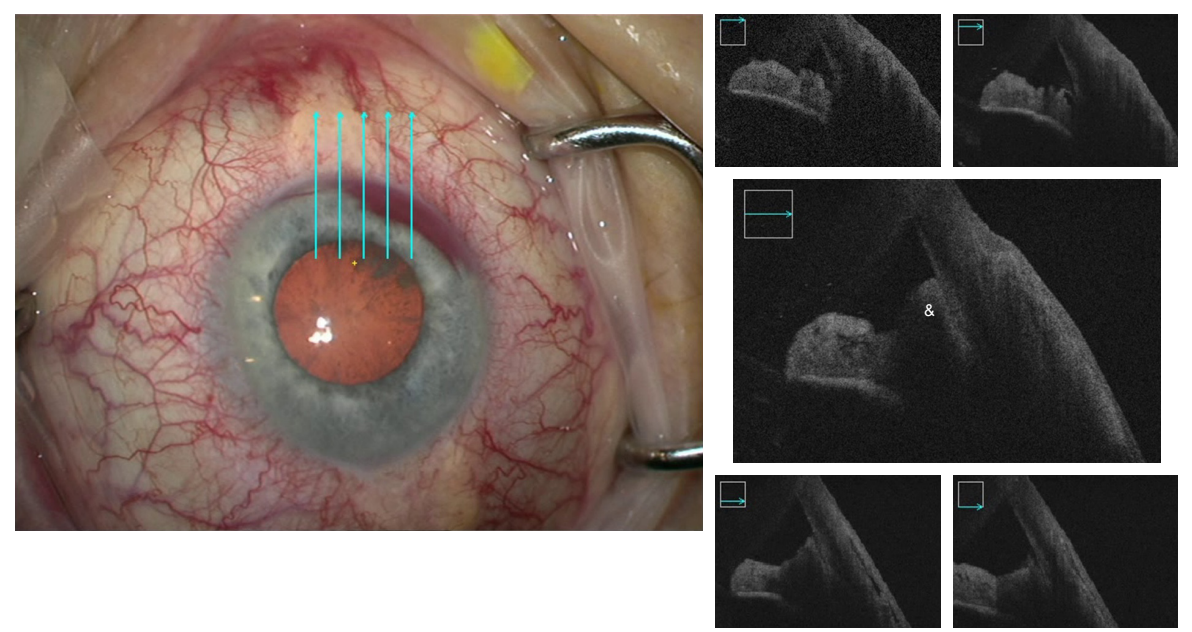

Figure I Color images: view from above through the OPMI LUMERA 700 surgical microscope (Carl Zeiss Meditec AG, Jena, Germany). Cyan arrows indicate the scan locations of the iOCT. Black and white images: corresponding iOCT scans of the ACA. (A) At the beginning of the surgery: *iris and ${ }^{\S}$ trabecular meshwork. (B) Postprocedure: >anterior part of the trabecular meshwork opening and \%area of Schlemm's canal. (C) Postprocedure: \&blood blocks the signal in the area of the trabecular meshwork opening.

Abbreviations: ACA, anterior chamber angle; iOCT, intraoperative optical coherence tomography.

\section{Discussion}

This is the first report to achieve an objective intraoperative monitoring of success using an iOCT in ab interno trabeculotomy with the Trabectome. The image quality can be nearly comparable to a standalone anterior segment OCT, but it is sometimes challenging to obtain good quality images. Using the modified Swan-Jacob lens, the ACA could be visualized quite well on the iOCT-scan and an objective assessment of 


\section{A}
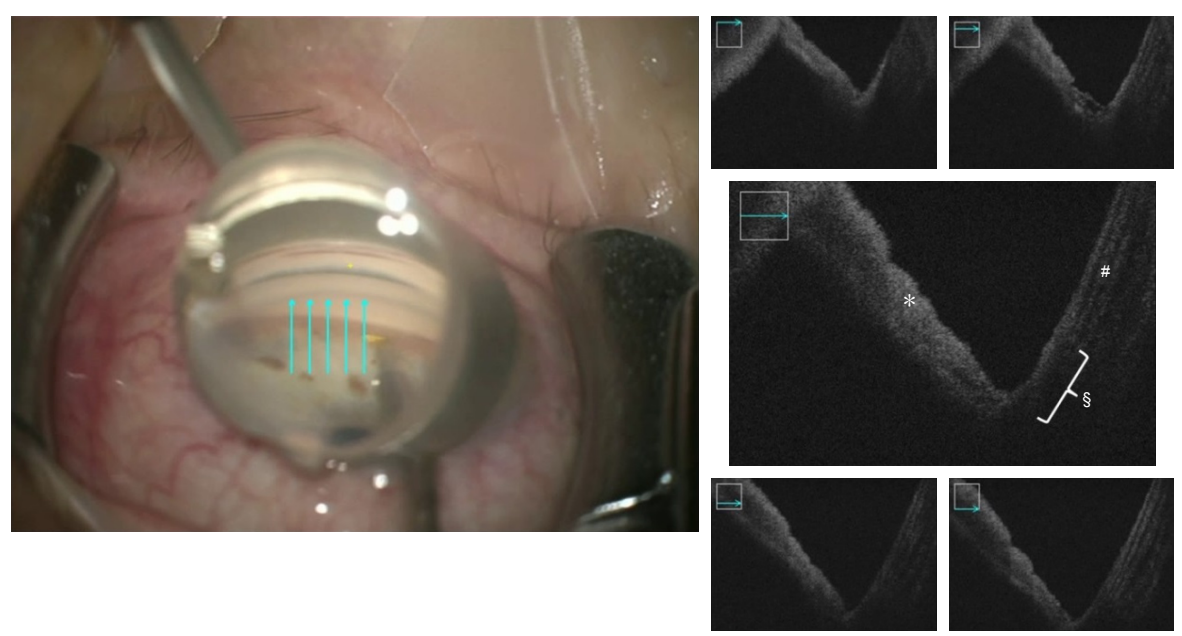

B
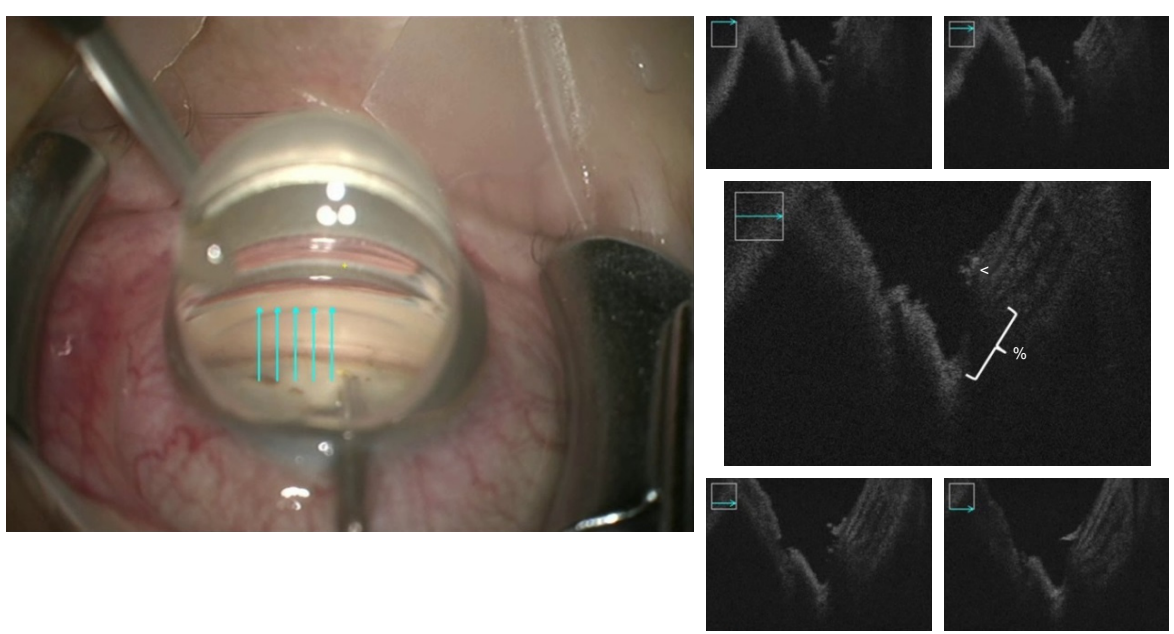

C
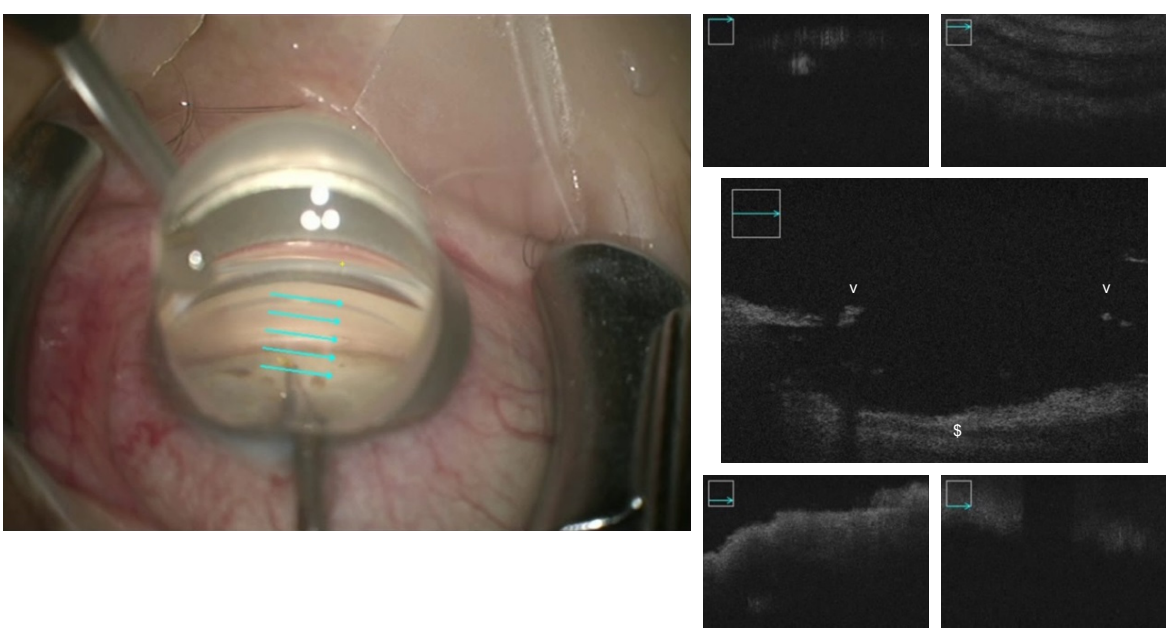

Figure 2 Color images: OPMI LUMERA 700 surgical microscope (Carl Zeiss Meditec AG, Jena, Germany) view through modified Swan-Jacob goniolens (Ocular Instruments, Bellevue, WA, USA) into the ACA. Cyan arrows indicate scan locations of the iOCT. Black and white images: corresponding iOCT scans of the ACA. (A) At the beginning

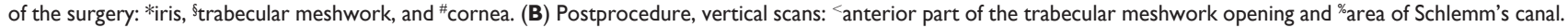
(C) Postprocedure, horizontal scans: 'border of the trabecular meshwork opening and 'scleral backwall of Schlemm's canal.

Abbreviations: ACA, anterior chamber angle; $\mathrm{IOCT}$, intraoperative optical coherence tomography.

the success of the surgical procedure, ie, the removal of the trabecular meshwork, could be achieved.

The handling of the iOCT is quite difficult. The surgeon can be in control of all iOCT functions via the foot panel of the OPMI, but then he/she cannot control the OPMI functions simultaneously. Therefore, it is best to have a technician who handles at least some of the iOCT functions via the touchscreen. 
One of the biggest problems is to focus on the region of interest (ROI). While it is relatively easy to get a stable iOCT scan of the retina and also (with minor difficulties) of the cornea and stay in focus during manipulation due to the inbuilt z-tracking, it is much harder to visualize the ACA. Due to the anatomical circumstances, the extent of the tissue in the area of the ACA in the $z$-axis is several millimeters thick, which can cause a mirroring effect in the iOCT scan due to the spectral-domain OCT technique. When it happens, it is difficult to stay in focus and to visualize the ROI due to the overlaying iOCT scan. One possibility to reduce this effect is using a short scan length. By reduction of scan length to a minimum of $3 \mathrm{~mm}$, we were able to gain best results without any disadvantages.

The second problem in the visualization of the ACA is the high reflectivity of the superficial layers that causes a shadowing effect on the underlying structures. Therefore, it was nearly impossible to get proper images using the direct view from above where the conjunctiva and the sclera reflect nearly all of the light.

We also tried to capture iOCT videos of the surgical process itself. Due to the constant change in focus level during the surgery while moving the instrument and the eye and the total reflectance of the metallic instrument, we did not succeed.

One major aspect in establishing the iOCT as a quality control tool in Trabectome surgery is the additional time. Since the Trabectome surgery normally lasts $<10$ minutes, the time needed to obtain useful images should be minimal. As expected, it took us a long time ( $\sim 15$ minutes) to obtain good quality images in our first patient. We had to learn how to handle the iOCT and how to find the right focus, and we examined six different scans (view from above pre-/postprocedural and view through modified Swan-Jacobs lens pre-/postprocedural with vertical and horizontal scan lines). For the last four patients, it took us only $2-4$ minutes to obtain the proper images (view through modified Swan-Jacob lens pre-/postprocedural with vertical scan lines $3 \mathrm{~mm}$ ). When we just want to objectify the immediate success of the surgical procedure, we need only the postprocedural scan that can be done in $<2$ minutes.

Heindl et $\mathrm{al}^{5}$ described the use of another iOCT device (OptoMedical Technologies, Luebeck, Germany) during ab interno trabeculotomy. They could show the positioning of the Trabectome tip inside the anterior chamber in one patient and the positioning of a trabecular aspiration cannula in another patient, but they did not report any pre- and/or postprocedural data. In their clinical setting, they could not visualize the whole ACA and the trabecular meshwork. Only in a setting with an experimental iOCT and in enucleated porcine eyes, they were able to show the trabecular meshwork.
Kumar et $\mathrm{al}^{6}$ recently reported the use of the Zeiss RESCAN 700 iOCT in four patients but no ab interno trabeculotomy. They also used this new technique as a quality control tool to intraoperatively check the positioning of the Ahmed tube and to monitor their surgical progress into the eye.

Siebelmann et $\mathrm{al}^{7}$ reported the use of the iOCT during canaloplasty in one patient. It seems to be a helpful device in monitoring the correct position of the suture and the change of the chamber angle after suture tensioning.

Further fields of application of an iOCT are during lamellar keratoplasty, where it seems to be more beneficial than in ab interno glaucoma surgery since it may support the surgeon throughout the whole procedure, ${ }^{8-12}$ and during vitreoretinal surgeries. ${ }^{13-18}$ These studies demonstrated that the use of an iOCT can change the surgical strategy in these procedures with potential benefits for the patients.

The limitations of our study are the small number of patients and the retrospective design.

\section{Conclusion}

To our knowledge, this is the first report of the use of the Zeiss RESCAN 700 iOCT for ab interno trabeculotomy with the Trabectome in a regular clinical setting. In spite of the described shortcomings of this new technique, we were able to visualize the ACA and to objectify the immediate success of the surgical procedure on iOCT scans, ie, an open gap in the trabecular meshwork. The iOCT can therefore be used as a quality control device in ab interno trabeculotomy.

\section{Acknowledgment}

The Zeiss RESCAN 700 was provided for 1 month without cost by Carl Zeiss Meditec AG.

\section{Disclosure}

BJ and CF received lecture fees from Carl Zeiss Meditec. The authors report no financial or proprietary interest in any material or method mentioned in the manuscript and no conflicts of interest in this work.

\section{References}

1. Jordan JF, Wecker T, van Oterendorp C, et al. Trabectome surgery for primary and secondary open angle glaucomas. Graefes Arch Clin Exp Ophthalmol. 2013;251(12):2753-2760.

2. Minckler DS, Baerveldt G, Alfaro MR, Francis BA. Clinical results with the trabectome for treatment of open-angle glaucoma. Ophthalmology. 2005;112(6):962-967.

3. Jacobi PC, Dietlein TS, Krieglstein GK. Microendoscopic trabecular surgery in glaucoma management. Ophthalmology. 1999;106(3): 538-544.

4. Dietlein TS, Engels BF, Jacobi PC, Krieglstein GK. UBM-guided chamber angle surgery for glaucoma management: an experimental study. Eye (Lond). 2003;17(3):340-345. 
5. Heindl LM, Siebelmann S, Dietlein T, et al. Future prospects: assessment of intraoperative optical coherence tomography in ab interno glaucoma surgery. Curr Eye Res. 2015;40(12):1288-1291.

6. Kumar RS, Jariwala MU, Sathidevi AV, et al. A pilot study on feasibility and effectiveness of intraoperative spectral-domain optical coherence tomography in glaucoma procedures. Transl Vis Sci Technol. 2015;4(2):2.

7. Siebelmann S, Cursiefen C, Lappas A, Dietlein T. Intraoperative optical coherence tomography enables noncontact imaging during canaloplasty. J Glaucoma. 2016;25(2):236-238.

8. Ehlers JP, Dupps WJ, Kaiser PK, et al. The prospective intraoperative and perioperative ophthalmic imagiNg with optical coherEncE tomogRaphy (PIONEER) study: 2-year results. Am J Ophthalmol. 2014; 158(5):999-1007.

9. Juthani VV, Goshe JM, Srivastava SK, Ehlers JP. Association between transient interface fluid on intraoperative OCT and textural interface opacity after DSAEK surgery in the PIONEER study. Cornea. 2014; 33(9):887-892.

10. Steven P, Le Blanc C, Lankenau E, et al. Optimising deep anterior lamellar keratoplasty (DALK) using intraoperative online optical coherence tomography (iOCT). Br J Ophthalmol. 2014;98(7):900-904.

11. Steven P, Le Blanc C, Velten K, et al. Optimizing descemet membrane endothelial keratoplasty using intraoperative optical coherence tomography. JAMA Ophthalmol. 2013;131(9):1135-1142.

12. Xu D, Dupps WJ, Srivastava SK, Ehlers JP. Automated volumetric analysis of interface fluid in descemet stripping automated endothelial keratoplasty using intraoperative optical coherence tomography. Invest Ophthalmol Vis Sci. 2014;55(9):5610-5615.
13. Ehlers JP, Itoh Y, Xu LT, Kaiser PK, Singh RP, Srivastava SK. Factors associated with persistent subfoveal fluid and complete macular hole closure in the PIONEER study. Invest Ophthalmol Vis Sci. 2015;56(2): 1141-1146.

14. Ehlers JP, Ohr MP, Kaiser PK, Srivastava SK. Novel microarchitectural dynamics in rhegmatogenous retinal detachments identified with intraoperative optical coherence tomography. Retina. 2013;33(7): 1428-1434.

15. Ehlers JP, Kernstine K, Farsiu S, Sarin N, Maldonado R, Toth CA. Analysis of pars plana vitrectomy for optic pit-related maculopathy with intraoperative optical coherence tomography: a possible connection with the vitreous cavity. Arch Ophthalmol. 2011;129(11):1483-1486.

16. Tao YK, Ehlers JP, Toth CA, Izatt JA. Intraoperative spectral domain optical coherence tomography for vitreoretinal surgery. Opt Lett. 2010; 35(20):3315-3317.

17. Junker B, Maier M, Agostini H, Hattenbach L-O, Pielen A, Framme C. [Intraoperative optical coherence tomography in retinal detachment]. Ophthalmologe. 2016;113(8):663-667.

18. Hattenbach L-O, Framme C, Junker B, Pielen A, Agostini H, Maier M. [Intraoperative real-time OCT in macular surgery]. Ophthalmologe. 2016;113(8):656-662.
Clinical Ophthalmology

\section{Publish your work in this journal}

Clinical Ophthalmology is an international, peer-reviewed journal covering all subspecialties within ophthalmology. Key topics include: Optometry; Visual science; Pharmacology and drug therapy in eye diseases; Basic Sciences; Primary and Secondary eye care; Patient Safety and Quality of Care Improvements. This journal is indexed on

\section{Dovepress}

PubMed Central and CAS, and is the official journal of The Society of Clinical Ophthalmology (SCO). The manuscript management system is completely online and includes a very quick and fair peer-review system, which is all easy to use. Visit http://www.dovepress.com/ testimonials.php to read real quotes from published authors. 